Uiloa $F$.

Rev. Chil. Pediatr. 64 (4); 272-276, 1993

\title{
Tentativas y consumación de suicidio en niños y adolescentes
}

\author{
Fresia Ulloa Ch.1
}

\section{Suicidal behaviour in children and adolescents}

Suicide is an important cause o death among adolescenl people. Suicidal attempls are more frequent, but resulting death is fairly less fiequent, at this age thon in ed:ts. Precipilating facters include social, family anc school ditticulties and also cxposure to suicidal behoviout by other people. Most employed self destiuctive methods are, in this courliy, oral neuroleptic and ansiolytic medications. Psychopathological significance of suicidal behaviour, personality aspocts, familial and social circumstances surrounding suicidal alternpts in children and adokscents are reviewed. Some immediate therapeutic measures are proposed and favourable condilions for appropriate long term care are described.

(Key words: suicidal atrempts, behoricur childien, adolescent.)

E1 suicidio está entre la segunda y tercera $(12 \%)$ de las causas de mucrte de jóvenes de 15 a 19 años de edad. Su incidencia se ha triplicado en los países occidentales en los últimos treinta anos, especialmente en adolcscentes y adultos jôvenes, pasando a constituir un problema importante de salud pública. Las causas del fenómeno no están claras, pero podrian estar ligadas a aumento del número de adolescentes en la población, que llevaría a disminución de las oportunidades de estudio, trabajo y buen éxito, generando baja autoestima. Otros factores pueden ser también inestabilidad familiar, violencia social, altas tasas de consumo de alcohol y drogas, depresión, mayor disponibilidad de armas de fuego y medicamentos en los hogares, transición más temprana de los jóvenes a roles adultos y disminución de la afiliación religiosa ${ }^{1-16}$.

\section{Epidemiología}

La prevalencia de la conducta suicida es diffcil de medir debido a falta de registros. Los jóve-

1. Médico psiquiatra infantojuvenil. Asesora de Salud Mental Infantojuvenil, Servicio Salud Metropolitano Norte. Hospital Roberto del Río, Depanamento de Pediatría, Campus None, Universidad de Chilc. nes en riesgo, aun después de un intento, rara vez consultan al médico $0^{2,4}$. En base a los datos disponibles, la tasa de tentativas autodestructivas a esta edad es mayor que en la población general, pero la letalidad es más baja, observándose una muerte cada 60 a 200 intentos en adolescentes y una cada 13 intentos en adultos $1,4,8,12,15$. La tasa anual de suicidios para todas las edades es de alrededor de 10 por 100000 habitantes, con diferencias importantes entre los distintos paises.

Las tentativas de suicidio ocurren más a menudo entre las mujeres que entre los hombres (3:1), lo que se asociaría con las presiones de socialización en niños y niñas, que se reflejan cn diferentcs estilos de expresión afectiva. Las mujeres en nucstra sociedad gozan de menor sanción cultural frente a las demostraciones de rabia 0 agresividad, incluyendo el suicidio. Tales diferencias parecen expresarse en la tendencia de las niñas a utilizar métodos más pasivos, a su vez menos efectivos, en su mejor disposición a buscar ayuda y más alta frecuencia de desórdenes afecLivos en la elapa postpuberal $1-7,12,14,15,17,18$, Los suicidios consumados son tres veces más frecucntes entre los varones, lo que pucde ser expljcado por la propensión de los jóvenes a utilizar medios más violentos e irreversibles.

Aunque la ideación suicida es frecuente en los niños menores de 12 años, las tentativas y el 
suicidio propiamente tal son $\operatorname{raros}^{2,6}, 10,15,18-21$, 10 que puede ser explicada en primer lugar, porque Ia enfermedad afectiva (depresiva), que es la causa más común de suicidio, es infrecuente a esta edad. En scgundo lugar, la familia, el colcgio y el ambiente provecn un entorno protector, con mayor apoyo emocional y social en esta etapa del desarrollo. En tercer término, para que el niño tenga sentimientos de desesperación y desesperanza, pucda planear y ejecutar un intento Ictal, se requicre cierto grado de mádurez cognitiva, que no se alcanza hasta la adolescen$\mathrm{cia}^{2}, 6,10,15$.

\section{Evolución del concepto de muerte}

La conducta suicida involucra el deseo de provocarse la mucrte. Sin embargo, la comprensión de la mucrte como el término irreversible de la vida no cstá prescnte en todos los niños y adolescentes suicidas. Lo importante es que la meta esencial de la conducta autodestructiva es lograr la muerte, independientemente de cómo la conceptualiza ese niño o adolescente en particular. El desarrollo del concepto de muerte es un proceso lento y prolongado $11,14,20,21$.

Según Ajuriaguerra, hay cuatro fases en la adquisición del concepto de muertc, las que se relacionan con el desarrollo cognitivo del nino. En la primcra, se observa incomprensión e indiferencia por el tcma, que sc prolonga hasta los 2 años de cdad. En la segunda, que dura hasta las edades de 466 años, hay una percepción mítica de la muerte, a su vez provisional, temporal y reversible; el niño acepta y niega simultáneamente sus consecuencias, atribuyéndole cualidades de vida. La tercera, es una fase concreta, de realismo y personificación, que se exticnde has. ta los 9 años. Para los niños, en esta etapa, la persona muere, pero permanece representable en cl tiempo y en espacio, está ausente, como petrificada en oura forma de vida. La cuarla fase es abstracta, se alcanza entre los 9 y 11 años de cdad. Aparece la angustia existencial, lo que supone acceso a la simbolización de la muerte, temor a la pérdida real y al final de su propio destino' ${ }^{19}$.

El conocimiento de este proceso permite comprender por qué aumenta la proporción de conductas destructivas en la adolescencia.

\section{Métodos utilizados}

Los niños y adolescentes llevan a cabo actos suicidas con diferentes métodos. Estos incluyen armas de fuego, medicamentos o venenos, ahorcamiento, salto desde altura, intoxicación con monóxido de carbono, Janzarniento al tráfico vehicular, incineración, asfixia por inmersión, apuñalamicnto $1,2,4-8,10,11,6,20,21$. En 20 casos de tentativas de suicidio en niños y adolescentes, atendidos en cl Hospital San Juan de Dios, de Santiago, Chile, cl método más utilizado fue la ingestión de medicamentos, especialmente ansiolílicos y neurolépticos ${ }^{18}$, lo que concuerda con publicaciones de otros paí$\operatorname{ses}^{5}, 7,8,20$, no así de Estados Unidos de Norteamérica, donde el más común son las armas de fuego ${ }^{2}$.

\section{Factores precipitantes}

Entre los factores precipitantes de conducta suicida en niños se describen los problemas de rendimiento y conducta cscolares, las discusiones con familiares o amigos, los desengaños amorosos, el abuso físico o sexual ${ }^{15}$. En los adolescentes sc mencionan las crisis disciplinarias y legales, pérdidas y conflictos interpersonales, exposición al suicidio o conducta suicida de lamiliares o amigos 2.6-16; acumulación de circunstancias vitales adversas, tales como cambios recientes de domicilio, partida de un miembro de la familia, cambios en los hábitos o formas de vida, ruptura con cl grupo de amigos ${ }^{3,2.6}$ $y$, en un pequeño número de casos, lecturas sobre suicidio en periódicos o novelas ${ }^{10}$. En Chile se han descrito, como factores precipitantes, situaciones de conflicto familjar, especialmente castigos y rcprensiones por parte de los padres, discusiones con los hermanos, restricción de permisos y amistades heterosexuales; mientras el fracaso escolar es un desencadenante menos frecuentc ${ }^{18}$. Alrededor de la mitad de las víctimas de suicidio consumado habian amenazado o intentado suicidio en las 24 horas previas a la muerte y un porcentaje similar habia faltado a la escuela el día anterior a su defunción ${ }^{10}$. En Estados Unidos sc observó quc los jóvenes suicidas frecuentemente cstaban intoxicados con alcohol al momento de morir ${ }^{6,16}$. 


\section{Significado de la conducta suicida}

El significado de la conducta suicida en los nifós es variable, puede representar una forma de evitar o huir de una situación desagradable o intolerable ${ }^{7,19,21}$, un intento de atraer la atención, obtener cariono 7.19 , pedir ayuda o castigo. Parece ser muy específico del niño cl deseo de una unión mágica, más allá de la muerte, con una persona que ha perdido o cree haber perdido $\left.{ }^{19}, 2\right]$. Entre los adolescentes, la mayoría de los intentos de suicidio son impulsivos $1,2,5,11,19$. Al interrogarlos acerca de los motivos que tuvieron para esta conducta, los identifican como una forma de huida o escape de una situación insoportable, expresión de duelo o desesperación por la pérdida de una persona significativa o la separación de sus padres; autocastigo, intención de influir en el otro, conseguir atención, comunicar amor, rabia, o sentimientos de revancha. En ocasiones puede ser expresión de un funcionamiento psicótico $1,2,5,7,11,17,22$.

\section{Características de los niños y adolescentes suicidas}

Este tipo de conductas se ve en niños con sintomas de depresión, rasgos de inmadurez. labilidad afectiva e impulsividad ${ }^{15,19,20}$. En un estudio, la mayoría de los niños afectados se encontraba dentro de alguna de las categorias de irritables y sensibles a la crítica; impulsivos e hiperactivos; pasivos, tranquilos, poco comunicativos y difíciles de entender; perfeccionistas, con alto nivel de exigencias y temerosos ante la posibilidad de cometer errores. Para Pfeffer ${ }^{20}$, Ios factores específicos que permiten distinguir a los niños suicidas de los no suicidas incluyen la presencia de depresión, de sentimientos de desesperanza y depreciación.

La mayoria de las tentativas de suicidio en adolescentes se presentan en ausencia de un trastorno psiquiátrico específico, siendo más bien expresión de problemas psicopatológicos del medio que rodea ai sujeto. Estos jóvenes muestran algunas características psicosociales disfuncionales, que incluyen sentimientos de desesperanza, dificultades en el control de impulsos, escasas habilidades sociales de resolución de problemas y modulación afectiva. La desesperanza ha sido correlacionada con la se- veridad del intento de suicidio. Sólo en 20 a $25 \%$ de estos casos se ha encontrado asociación entre ientativas autodestructivas y trastornos psiquiátricos, tales como desordenes afectivos (depresión uni o bipolar), conducta antisocial y abuso de alcohol y drogas. Sólo una minoría tiene problemas de adaptación y rendimiento esco$\operatorname{lar}^{1,2,5,6,9,11,12,16,22 .}$

\section{Circunstancias familiares $y$ tentativas de suicidio}

En las familias de estos niơos y adolescentes sucle existir inestabilidad y desorganización. Entre los conflictos familiares destacan especiatmente las separaciones y la ausencia del padre u otras figuras parentales. La actitud afectiva de los padres puede ser ambivalente, con aceptación y hostilidad o rechazo. Estos niños y adolescentes han cstado expuestos, frecuentemente, a vioIencia familiar y han sido victimas de abuso físico o sexual $-2,5-13,15,18,20 \cdot 22$. La falta de comunicación que existe entre padres e hijos dificulta el acercamiento y la solicitud de ayuda por parte del sujeto que enfrenta conflictos que no pucde resolver ${ }^{8,18}$.

Suele haber antecedentes familiares de intentos de suicidio y suicidios consumados; de enfermedades psiquiátricas, especialmente alcoholismo del padre o de la madre, desordenes afectivos y desórdenes antisociales $1,2,6,9-11,13,15,20$. Esta conducta no se hereda, pero es mucho más frecuente en familias en que un componente se ha suicidado, to que no sólo aumenta el riesgo para los niños, sino también para los otros miembros de la familia2, 10, 11, 56 .

\section{Reincidencia}

El individuo que ya ha realizado una tentativa de suicidio tiene alta probabilidad de repetirla y se estima que 30 a $50 \%$ de los casos reinciden. El riesgo es mayor en los tres meses que siguen al intento inicial; en sujetos de mayor edad (sobre los 13 años); cuando el primer intento ha sido potencialmente letal y premeditado; en personas con algún desorden psicopatológico o antecedentes de dificultades de adaptación o rendimiento escolar; miembros de familia numerosa, de 4 niños o más, con antecedentes de alcoholismo, 
abuso y disfunción familiar; niños que no cumplen el tratamiento $1,2,6,10,12,1 \leq, 20$.

En un seguimicnto de 10 a 15 años de duracion, de adolescentes y adultos jovenes que habían prescntado conducta suicida, 2,9\% de las mujeres y $10 \%$ de los varones muricron por suicidio en período scñalado. La elapa de mayor ricsgo, en esta muestra, fueron los dos primeros años después del intento inicial's. En una experiencia chilena se ha observado que, en el momento de la primera consului. 40\% de los pacientes estudiados tenían antecedentes de tentativas previas y la mitad de eltos más de una $^{18}$.

\section{Medidas terapéuticas}

Las medidas terapécuticas deben ser inmediatas, como respuesta directa al acto suicida. Se recomienda no dar de alta directamente al paciente desde el servicio de urgencia, dejándolo al menos $48 \mathrm{~h}$ en el hospital, lo que da tiempo para establecer una relación con él y evaluarlo, como también a su familia

El objetivo más importante de esta evaluación es precisar la magnitud del peligro inmediato del paciente. Para esto es necesario identificar las circunstancias que rodean la idcación o acto suicida, el método utilizado, la existencia de premeditación y planificación y las posibilidades que tenía de ser descubierto y rescatado 4.25 . Existc una escala de evaluación de ricsgo-rcscate, basada en la premisa que todos los intentos de suicidio reflcjan ambivalencia, es decir, un desco de morir y de ser rescatado simultáncamentc. A mayor cvidencia en favor del deseo de morir y no ser rescatado, mayor es el riesgo de reincidencia y muerte $^{5,12,23}$. La hospitalización permite, también, scparar al niño o adolescente de su vida cotidiana, lo que facilita cl control de los factores que han desencadenado la conducta autodestructiva. Es importante conseguir un acucrdo con el paciente (contrato no suicida) en el que éste se compromete a frenar sus impulsos autodestructivos y comunicarlos al médico u otro adulto2,6. Hablar sobre la idcación suicida no parece promover más ideas o actos de la misma naturaleza. Las preguntas sobre el tema debcrían ser directas y no implícitas. Esto constituye el primer paso en la intervención terapéutica ${ }^{5}, 12,20$. En todo paciente se debe solicilar una inter- consulta psiquiátrica para cl examen de su estado mental.

El médico pediatra desempeña un importante papel en el cumplimiento de las indicaciones de tratamiento. Cerca de $50 \%$ de los pacientes no concurren a la primera consulta anbulatoria y sólo 32\% completan tres sesioncs ${ }^{2}$. Para evitar estas fallas es útil que el pediatra que hizo la cvaluación inicial mantenga el vínculo con $\mathrm{cl}$ paciento y sca quicn to presente al profesional que va a continuar la terapia.

Las cilaciones at especialista deben ser definidas desde el comienzo (lugar, día y hora) $y$, dentro de lo posible, el paciente debe ser atendido precozmente (dentro de las primeras 48 horas). Se debe cvitar reaccionar con agresividad frente a la inasistencia de ćste y de sus familiares. Un aspecto fundamental del mancjo es ponerse de acuerdo con el pacicnte y la familia sobre el tipo y la duración del uatamiento que ellos necesitan y aceptan. Es úlil involucrar en la terapia a otras personas significalivas de la familia, escucla y amigos $^{2.5}$. La hospitalización psiquiátrica está indicada si no hay posibilidad de establecer un contrato no suicida, la ideación suicida es activa, cl intento ha sido potencialmente letal, hay un trastomo psicopatológico imporlante (psicosis, desorden depresivo o bipolar, abuso de sustancias, uastornos de conducta), no se cumple o fracasa cl mancjo ambulatorio o existe disfunción familiar severa.

El tratamicnto debe ser integral y, dependiendo de la condición específica de cada paciente, se utilizarán psicofármacos, psicoterapia individual y familiar, $11,23,24$ por el ticmpo que sea necesario. Pueslo que la ideación suicida y las conductas autodestruclivas son frecuentes en niños y adolescentes, es de gran importancia que los médicos intcroguen sistemáticamentc sobre eslos aspectos a los pacicntes con ricsgo: aquellos en que se sospechan problemas psiquiátricos, que tienen antecedentes de mala adaptación social, historia de pérdidas interpersonales, problemas familiares (disfunción y afecciones psiquiálricas) y cxposición a suicidio.

\section{Resumen}

Las tentativas de suicidio y el suicidio consumado constituyen una de las principales causas de muerte en adolescentes. Se hace una revision 
del tema, destacando los aspectos epídemiologicos, factores precipitantes, métodos autodestructivos utilizados, significado psicopatológico de la conducta, características de los niños y adolescentes suicidas y circunstancias familiares que rodean al hecho. Se proponen algunas medidas terapéuticas inmediatas, frente al acto suicida, y se describen las condiciones que favorecen el tratamiento espccializado posterior.

(Palabras clave: tentativas de suicidio, suicidio, niños y adolescentes.)

\section{Referencias}

1. Marcelli D, Braconmier A: Las tentativas de suicidio. En: Marcelli D, Braconnier A. Manual de Psicopatología del Adolescentc, Barcelona, Editorial Masson, 1986; 99-1 I4.

2. Brent $B, K$ olko $D$. Suicide and suicidal behaviour in children and sdolescents, En: Garfinkel B, ed. Psychiatric Disorders in Children and Adolescents, I'hila. de]phia, Lditorial Saunders Company, 1990; 372-391.

3. Eisenberg $L$ : The cpidemiology of suicide in adolescents. Pediatr Ann 1984; 13: 47-53.

4. Casorla $R$ : Suicidio e adolesccncia. I.- Incidencia, histórico e fatores suicidogenos. Acta Psiquiatr Psicol Am 1.a1 1979: 25: 288-295.

5. Alonso-Fernández F. Conducla suicida en la adolescencia. Psicopatología 1985: 5: 147-159.

6. Brent D: Suicide and suicidal behavior in children and adoicscents. Pediate Rev 1989; 10: 269-275.

7. Vefilla Picazo $M$ : La lentativa de suicidio en la adoJescencia. Comunicación Psiquiátrica. 1980: $21-59$.

8. Svedo $S$, Rutsew D, Kuppenheimer M. Lum D, Dolan S, Goldenberg $E$ : Can adolescents suicide auempters be distinguished from at risk adolescents? Pedialrics 1991; 88: 620-629.

9. Turgay A: An integrative trcaument approach to child and adolescent suicidal behavior. Psychiatr Clin North Am 1989; 12: $971-985$.
10. Shaffer D: Suicide. En: Rutuer M, Fersov L, ed. Child and Adolescent Psychiatry, Oxford: Blackwell Scicntific Publications, 1985; 708-719.

11. Pfeffer $C$ : Atlempted suicide in children and adolesconts: causes and management. En: Lewis $M$, ed.: Child and Adolescent Psychiatry. A comprehensive textbook. Baltimore: Williams and Wilkins, 1991: 664. 672.

12. Hergenroeder A, Kastner L. Farrow J, Deisher $R$ : The pcdiatrician's role in adolescent suicide. Pediatr Ann 1986: 15: 787-798.

13. Drake $R$, Racusin $R$, Murphy $T$ : Suicide among adolesecnts with mentally ill parents. Hosp Community Psychiatry 1990; 41: 921-922.

14. Fundufis $T$ : Mood disorders, suicide and suicide bchavıor. Cur Opin Pediatr 1990; 2: 700-704.

15. Jiménez $J$, Barrón E. Pérez de Heredia $J$, Sastre $M$. Intentos de suicidio en la infancia. Revista Española Pedialría 1991; 47: 405-409.

16. Pfeffer C. Assessment of suicidal children and adolescents. Psychiatr Clin North Am 1989; 12: 861872 .

17. Sáz A. Las tentativas de suicidio del niño y del adolescente. Actas Luso Isp Neurol Psiquiatr Cienc Afines 1985; 13: 77-82.

18. Roma $E$. Wettlin $F$ : Tentativas de suicidio en niños y adiolescentes. Rev Chil Pediatr 1986; 57: 227-230.

19. Ajuriaguerra $J$, Marcelli $D$ : Tentativas de suicidio en cl niño. Fn: Ajuriaguerra J, ed. Manual de Psicopatología del Niño. Barcelona: Toray-Masson, 1987; 180 189.

20. Pfeffer $C$ : Clinical aspects of childhood suicidal behavior. Pedialr Ann 1984; 13: 56-61.

21. Krinsky $S$ : La conducla suicida en la infancia. Psicopátologia 1985; $5: 137-142$

22. Laborde de Moya J: Tentativas de suicidio en menotcs de 16 años. Neuropsiquiatría y Salud Mental (Arg) 1981; XII 1-96: 42-48.

23. Nucelse-Rios: Prcvención del suicidio en adolescentes. Niños 1988; 23: 110-118.

24. Pfeffer C, Newcorn J, Kaplan G, Mizruchi M. Plutchik $R$ : Sublypes of suicidal and assaultive behaviors in adolescents psychiatric inpatients: a research note. I Child Psychol Psychiatry 1989; 30: 151-163. 